OPEN ACCESS

Edited by:

Gianmarco Contino,

University of Cambridge,

United Kingdom

Reviewed by:

Ben George,

Medical College of Wisconsin,

United States

Douglas Evans,

Medical College of Wisconsin,

United States

Helmut Friess,

Technical University of

Munich, Germany

*Correspondence:

Kerrington D. Smith

kerrington.d.smith@hitchcock.org

Specialty section:

This article was submitted to

Gastrointestinal Cancers,

a section of the journal

Frontiers in Oncology

Received: 16 March 2020

Accepted: 09 July 2020

Published: 16 September 2020

Citation:

Trinh KV, Fischer DA, Gardner TB and

Smith KD (2020) Outcomes of

Neoadjuvant Chemoradiation With and Without Systemic Chemotherapy

in Resectable and Borderline

Resectable Pancreatic

Adenocarcinoma.

Front. Oncol. 10:1461.

doi: 10.3389/fonc.2020.01461

\section{Outcomes of Neoadjuvant Chemoradiation With and Without Systemic Chemotherapy in Resectable and Borderline Resectable Pancreatic Adenocarcinoma}

\author{
Katherine V. Trinh ${ }^{1}$, Dawn A. Fischer ${ }^{2}$, Timothy B. Gardner ${ }^{1}$ and Kerrington D. Smith ${ }^{2 *}$ \\ ${ }^{1}$ Department of Gastroenterology and Hepatology, Dartmouth-Hitchcock Medical Center, Lebanon, NH, United States, \\ ${ }^{2}$ Department of General Surgery, Dartmouth-Hitchcock Medical Center, Lebanon, NH, United States
}

Introduction: Neoadjuvant therapy is increasingly being used for localized pancreatic adenocarcinoma. While there is evidence supporting neoadjuvant systemic chemotherapy as well as chemoradiation, more evidence is needed to determine whether systemic chemotherapy with chemoradiation offers benefits over chemoradiation alone. This study compares the outcomes of neoadjuvant chemoradiation therapy with and without systemic chemotherapy in resectable and borderline resectable pancreatic cancers.

Methods: This retrospective study evaluated patients with resectable and borderline resectable pancreatic adenocarcinoma who completed neoadjuvant chemoradiation therapy with and without systemic chemotherapy prior to surgical resection. 149 patients met inclusion criteria, with 75 having resectable cancer and 74 having borderline resectable cancer. Outcomes included recurrence free and overall survival rates at 6 , 12 , and 36 months.

Results: In resectable pancreatic carcinoma, $72 \%$ of patients treated with chemoradiation alone achieved 1 year recurrence free survival compared to $78 \%$ of patients treated with systemic chemotherapy and chemoradiation $(p=0.55) .28 \%$ of patients treated with chemoradiation alone had 3 years recurrence free survival compared to $31 \%$ of patients who received systemic and chemoradiation therapy $(p=0.75)$. In both treatment groups, $92 \%$ of patients lived past 1 year $(p=0.92)$, and $44 \%$ of patients survived at least 3 years $(p=0.95)$. In borderline resectable pancreatic carcinoma, 50\% of patients treated with chemoradiation alone achieved 1 year recurrence free survival compared to $70 \%$ of patients treated with systemic chemotherapy and chemoradiation $(p=0.079)$. The 3 years recurrence free survival was 26 and $29 \%$ for the chemoradiation alone group and the systemic chemotherapy plus chemoradiation group, respectively $(p=0.85)$. There was no significant difference in 1 year overall survival: $85 \%$ of patients treated with chemoradiation alone survived compared to $92 \%$ of patients treated with 
systemic chemotherapy and chemoradiation $(p=0.32)$. Both groups had $41 \% 3$ years overall survival $(p=0.96)$.

Discussion: In resectable and borderline resectable pancreatic adenocarcinoma, there was no significant difference in overall or recurrence free survival between patients treated with chemoradiation with and without systemic chemotherapy. Our findings suggest that systemic neoadjuvant chemotherapy with chemoradiation and chemoradiation alone are efficacious treatments for localized pancreatic carcinoma. This brings into question whether more effective systemic chemotherapy is necessary to increase survival benefit.

Keywords: pancreas-adenocarcinoma, borderline resectable, neoadjuavant chemotherapy, chemoradiation (CRT), pancreatic cancer, systemic chemotherapy

\section{INTRODUCTION}

Pancreatic cancer is the tenth most commonly diagnosed malignancy and fourth leading cause of cancer related mortality in the United States (1). Surgical resection has been considered the only curative treatment modality, however treatment options include surgical resection alone, surgical resection followed by adjuvant therapy, and neoadjuvant therapy prior to surgical resection with or without adjuvant therapy. The neoadjuvant approach has gained increasing popularity in the last decade despite controversy over its risks and benefits. Neoadjuvant therapy offers the theoretical advantages of downstaging borderline resectable or locally advanced tumors, enabling more patients to be candidates for surgical resection, increasing the rate of margin negative resection, treating occult micrometastatic disease, optimizing selection of surgical candidates, and increasing overall survival (2-4). Conversely, neoadjuvant therapy in pancreatic adenocarcinoma has variable response rates and delaying surgical resection may risk missing the opportunity for the only potentially curative modality $(4,5)$.

Standard definitions for resectability designation were created in an effort to standardize patients into groups based on likelihood and potential for margin negative resection. The AHPBA/SSAT/SSO guideline uses objective CT imaging criteria to designate pancreatic adenomas as resectable, borderline resectable, locally advanced, and metastatic (6). In addition to defining the role of neoadjuvant therapy in resectable and borderline resectable pancreatic cancer, more data is needed to compare different neoadjuvant regimens, including the use of systemic chemotherapy (SCT), chemoradiation therapy (CRT), or both. Several studies have demonstrated decreased rates of local recurrence and improved survival outcomes using various regimens in the preoperative setting, however more evidence is needed to elucidate the optimal treatment protocol $(7,8)$.

Greer et al. demonstrated that neoadjuvant gemcitabinebased CRT decreased rates of local recurrence in pancreatic adenocarcinoma (9). The PRODIGE 4/ACCORD 11 and MPACT trials demonstrated improved survival outcomes in patients with metastatic disease using neoadjuvant SCT with FOLFIRINOX and gemcitabine/abraxane over gemcitabine alone. It is still unknown whether similar results are observed in localized, nonmetastatic pancreatic adenocarcinomas $(10,11)$. A recent study by Jang et al. found that gemcitabine based neoadjuvant CRT offered survival benefits over up front surgery in patients with borderline resectable pancreatic adenocarcinoma. This clinical trial not only demonstrated the efficacy of neoadjuvant therapy in borderline resectable disease, but also the efficacy of gemcitabinebased CRT in the absence of SCT (12).

Thus, while there is data supporting both SCT with and without CRT, as well as CRT alone, more evidence is needed to determine what the optimal neoadjuvant protocol entails in the setting of resectable and borderline resectable cancer. In this study, we compiled 13 years of data to compare the outcomes

TABLE 1 | Classification of neoadjuvant regimens into radiation-sensitizing agents and systemic chemotherapy agents.

\section{RADIATION-SENSITIZING REGIMENS \\ Gemcitabine \\ $50 \mathrm{mg} / \mathrm{m}^{2}$ of gemcitabine with radiation therapy twice weekly for 12 doses with a total of 50.4 Gy \\ Gemcitabine/Cetuximab $\quad 400 \mathrm{mg} / \mathrm{m}^{2}$ cetuximab loading dose 1 week prior to radiation followed by $250 \mathrm{mg} / \mathrm{m}^{2}$ cetuximab once a week for 6 doses in addition to $50 \mathrm{mg} / \mathrm{m}^{2}$ of gemcitabine with radiation therapy twice weekly for 12 doses with a total of 50.4 Gy}

\section{SYSTEMIC CHEMOTHERAPY AGENTS*}

Gemcitabine/Docetaxel

$65 \mathrm{mg} / \mathrm{m}^{2}$ docetaxel and $400 \mathrm{mg} / \mathrm{m}^{2}$ gemcitabine every 2 weeks for 3 doses

Gemcitabine/Abraxane

$1,000 \mathrm{mg} / \mathrm{m}^{2}$ gemcitabine and $125 \mathrm{mg} / \mathrm{m}^{2}$ abraxane weekly; patients underwent $2-3$ cycles over the span of 6-12 weeks depending on patient response; the average duration was 9 weeks

FOLFIRINOX

$$
\begin{aligned}
& \text { Biweekly } 85 \mathrm{mg} / \mathrm{m}^{2} \text { oxaliplatin, } 400 \mathrm{mg} / \mathrm{m}^{2} \\
& \text { leucovorin, } 180 \mathrm{mg} / \mathrm{m}^{2} \text { irinotecan, and } 400 \mathrm{mg} / \mathrm{m}^{2} \\
& \text { fluorouracil bolus followed by } 2,400 \mathrm{mg} / \mathrm{m}^{2} \\
& \text { fluorouracil via continuous infusion over } 26 \mathrm{~h} \text {; } \\
& \text { patients underwent } 4-5 \text { cycles over the span of } \\
& \text { 8-20 weeks depending on patient response; the } \\
& \text { average duration was } 12 \text { weeks. }
\end{aligned}
$$

${ }^{*}$ All systemic chemotherapy protocols include subsequent chemoradiation therapy with $50 \mathrm{mg} / \mathrm{m}^{2}$ of gemcitabine with intensity modulated radiation therapy twice weekly for 12 doses, with a total IMRT radiation dose of $50.4 \mathrm{~Gy}$. 
of neoadjuvant CRT with and without SCT in resectable and borderline resectable pancreatic adenocarcinoma. In addition to the previous most commonly used regimen of gemcitabine-based CRT alone and the currently popular systemic regimens of FOLFIRINOX and gemcitabine/abraxane, this retrospective study includes our institution's experience with two other neoadjuvant protocols: radio-sensitizing gemcitabine/cetuximab and systemic gemcitabine/docetaxel.

\section{METHODS}

\section{Study Design, Patient Selection}

This study was approved by the Dartmouth Committee for the Protection of Human Subjects. We performed a single-institution retrospective study on patients at Dartmouth-Hitchcock Medical Center from 2004 to 2017. Selection criteria included patients who were reviewed by our institution's multidisciplinary gastrointestinal tumor board with histologic diagnoses of

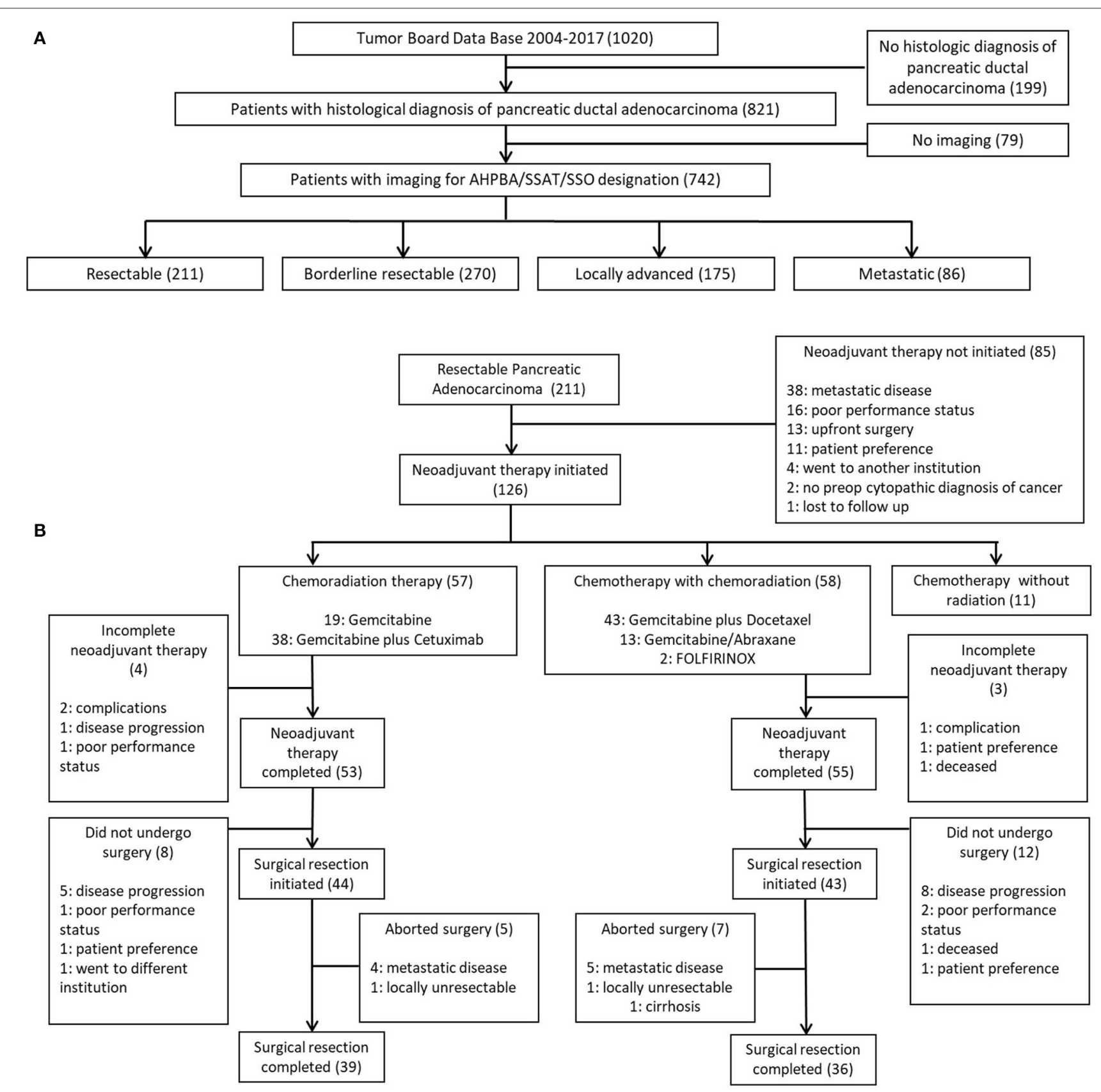

FIGURE 1 | Continued 


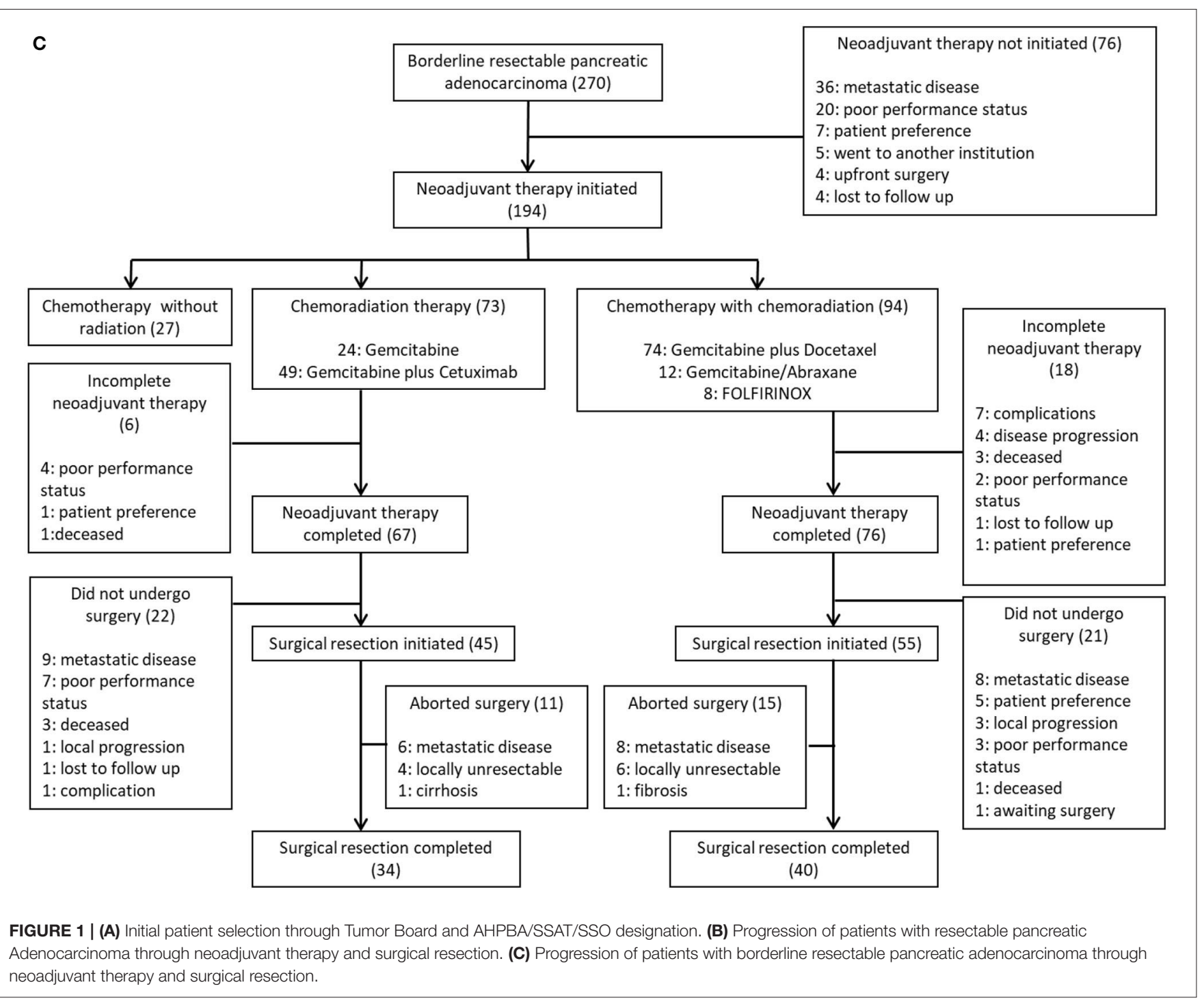

pancreatic adenocarcinoma and imaging for resectability designation. All CT scan images were reviewed retrospectively and categorized according to the AHPBA/SSAT/SSO resectability designation. Only cancers with resectable or borderline resectable designations were included in our study. Resectable tumors were defined as a primary tumor with an intact tissue plane (no venous or atrial abutment) between the tumor and the superior mesenteric vein (SMV), portal vein (PV), superior mesenteric artery (SMA), and common hepatic artery. Borderline resectable tumors were defined as radiographic evidence of tumor-associated deformity of the SMV or PV, abutment of the SMV or PV $>180^{\circ}$, short-segment occlusion of the SMV or PV amenable to resection and reconstruction, short-segment involvement of the hepatic artery or its branches amenable to resection and reconstruction, or abutment of the SMA $<180^{\circ}$ (6).

\section{Neoadjuvant Protocols}

Our two treatment groups were composed of patients who received neoadjuvant radiation-sensitizing CRT alone and patients who received neoadjuvant SCT and CRT. The CRT only regimens included intensity modulated radiation therapy (IMRT) with concurrent gemcitabine alone and gemcitabine/cetuximab. The gemcitabine alone protocol consisted of $50 \mathrm{mg} / \mathrm{m}^{2}$ of gemcitabine with radiation therapy twice weekly for 12 doses with a total dose of 50.4 Gray. The gemcitabine/cetuximab protocol consisted of a loading dose of $400 \mathrm{mg} / \mathrm{m}^{2}$ cetuximab intravenously 1 week prior to radiation followed by $250 \mathrm{mg} / \mathrm{m}^{2}$ cetuximab intravenously once a week for 6 weeks in addition to $50 \mathrm{mg} / \mathrm{m}^{2}$ of gemcitabine with radiation therapy twice weekly with a total dose of 50.4 Gray.

The SCT regimens included gemcitabine/docetaxel, gemcitabine/abraxane, and FOLFIRINOX, which were 
sequenced upfront at the time of diagnosis prior to CRT. The gemcitabine/docetaxel regimen included $65 \mathrm{mg} / \mathrm{m}^{2}$ docetaxel and $400 \mathrm{mg} / \mathrm{m}^{2}$ gemcitabine intravenously every two weeks for three doses. The gemcitabine/abraxane regimen consisted of $1,000 \mathrm{mg} / \mathrm{m} 2$ gemcitabine and $125 \mathrm{mg} / \mathrm{m}^{2}$ abraxane weekly. Patients underwent 2-3 cycles over a period of 612 weeks (average 9 weeks) depending on patient response. FOLFIRINOX included biweekly $85 \mathrm{mg} / \mathrm{m}^{2}$ oxaliplatin, 400 $\mathrm{mg} / \mathrm{m}^{2}$ leucovorin, $180 \mathrm{mg} / \mathrm{m}^{2}$ irinotecan, and $400 \mathrm{mg} / \mathrm{m}^{2}$ fluorouracil bolus followed by $2,400 \mathrm{mg} / \mathrm{m}^{2}$ fluorouracil via continuous infusion over $26 \mathrm{~h}$. Patients underwent $4-5$ cycles over a period of 8-20 weeks (average 12 weeks) depending on patient response. All SCT protocols included subsequent CRT with $50 \mathrm{mg} / \mathrm{m}^{2}$ of gemcitabine with radiation therapy twice weekly for 12 doses, with a total IMRT dose of 50.4 Gray. Table 1 lists all regimens used and their classification into SCT with CRT, and radiation-sensitizing CRT alone.

\section{Patient and Tumor Characteristics}

We obtained baseline characteristics including patient age, gender, Carbohydrate antigen 19-9 (CA 19-9) levels, endobiliary stent presence, tumor size, tumor location, $\mathrm{T}$ classification, and $\mathrm{N}$ classification. We also collected data on neoadjuvant regimen and completion rate. Information on whether patients made it to surgical resection, whether surgical resection was completed or aborted, and whether patients received adjuvant therapy following surgical resection was also obtained.

TABLE 2 | Baseline characteristics for patients with resectable pancreatic adenocarcinoma.

\begin{tabular}{|c|c|c|c|}
\hline Variable & $\begin{array}{c}\text { Chemoradiation } \\
\qquad n=39\end{array}$ & $\begin{array}{c}\text { Chemotherapy \& } \\
\text { chemoradiation } \\
n=36\end{array}$ & $p$-value \\
\hline $\begin{array}{l}\text { Age at diagnosis, mean (SD), } \\
\text { years }\end{array}$ & $66(10.2)$ & $67.2(9.2)$ & 0.59 \\
\hline \multicolumn{4}{|l|}{ GENDER (\%) } \\
\hline Female & $18(46)$ & $13(36)$ & 0.38 \\
\hline Male & $21(54)$ & $23(64)$ & \\
\hline \multicolumn{4}{|l|}{ ENDOBILIARY STENT (\%) } \\
\hline Yes & $28(72)$ & $20(56)$ & 0.14 \\
\hline No & $11(28)$ & $16(44)$ & \\
\hline \multicolumn{4}{|l|}{ TUMOR LOCATION (\%) } \\
\hline Head & $30(77)$ & $26(72)$ & 0.64 \\
\hline Neck, body, or tail & $9(23)$ & $10(28)$ & \\
\hline Tumor diameter, mean (SD), cm & $2.76(1.01)$ & $2.68(0.75)$ & 0.71 \\
\hline CA 19-9, mean (SD) & 298 (673) & $571(1,393)$ & 0.29 \\
\hline \multicolumn{4}{|l|}{ T CLASSIFICATION (\%) } \\
\hline TO & $2(5)$ & $2(6)$ & 0.79 \\
\hline T1 & $6(15)$ & 7 (19) & \\
\hline T2 & $3(8)$ & $1(3)$ & \\
\hline T3 & $28(72)$ & $26(72)$ & \\
\hline \multicolumn{4}{|l|}{ N CLASSIFICATION (\%) } \\
\hline No & $31(79)$ & $29(81)$ & 0.57 \\
\hline N1 & $8(21)$ & $7(19)$ & \\
\hline
\end{tabular}

\section{Outcome Measures}

Intention to treat survival analysis was done on all patients who underwent neoadjuvant therapy, regardless of whether they completed their regimen or made it to surgical resection. A separate analysis included only patients who completed neoadjuvant therapy and surgical resection. We collected information on resection margins and recurrence patterns. R0 resection is defined as tumor $>1 \mathrm{~mm}$ away from margins, and R1 resection is defined as tumor $<1 \mathrm{~mm}$ away from margins. Local recurrence was defined as any soft tissue density that was new or increased in size compared to prior images within the resection bed, regional lymph nodes, or at the pancreaticojejunostomy or biliary anastomoses. Distant metastatic recurrence was defined as new masses outside of the surgical bed, regional draining lymph nodes, or anastomoses. Distant metastases were categorized by number and organ site. Overall survival and recurrence free survival data were collected for 6 months, 1 year, and 3 years. Both overall and recurrence free survival were calculated from date of diagnosis.

\section{Statistical Analysis}

Continuous variables representing patient baseline characteristics and outcomes were represented as the mean and standard deviation. Categorical data were summarized as frequency and percentage. Continuous variables were analyzed using the Student's $t$-test, and categorical variables were

TABLE 3 | Baseline characteristics for patients with borderline resectable pancreatic adenocarcinoma.

\begin{tabular}{|c|c|c|c|}
\hline Variable & $\begin{array}{l}\text { Chemoradiatio } \\
\qquad n=34\end{array}$ & $\begin{array}{c}\text { Chemotherapy \& } \\
\text { chemoradiation } \\
\qquad n=40\end{array}$ & $p$-value \\
\hline $\begin{array}{l}\text { Age at diagnosis, mean (SD), } \\
\text { years }\end{array}$ & $62.7(8.4)$ & $63.8(8.5)$ & 0.56 \\
\hline \multicolumn{4}{|l|}{ GENDER (\%) } \\
\hline Female & $16(47)$ & $20(50)$ & 0.80 \\
\hline Male & $18(53)$ & $20(50)$ & \\
\hline \multicolumn{4}{|l|}{ ENDOBILIARY STENT (\%) } \\
\hline Yes & $28(82)$ & $29(72)$ & 0.32 \\
\hline No & $6(18)$ & $11(28)$ & \\
\hline \multicolumn{4}{|l|}{ TUMOR LOCATION, (\%) } \\
\hline Head & $31(91)$ & $31(78)$ & 0.11 \\
\hline Neck, body, or tail & $3(9)$ & $9(22)$ & \\
\hline Tumor diameter, mean (SD), cm & $3.97(4.34)$ & $3.25(1.61)$ & 0.33 \\
\hline CA 19-9, mean (SD) & $576(1,738)$ & $443(713)$ & 0.69 \\
\hline \multicolumn{4}{|l|}{ T CLASSIFICATION (\%) } \\
\hline TO & $3(9)$ & $0(0)$ & 0.057 \\
\hline T1 & $5(15)$ & $1(3)$ & \\
\hline T2 & $0(0)$ & $2(5)$ & \\
\hline T3 & $24(71)$ & $34(85)$ & \\
\hline T4 & $2(6)$ & $3(8)$ & \\
\hline \multicolumn{4}{|l|}{ N CLASSIFICATION (\%) } \\
\hline NO & $27(79)$ & $26(65)$ & 0.13 \\
\hline N1 & $7(21)$ & $14(35)$ & \\
\hline
\end{tabular}


analyzed using the chi-squared test and Fisher's exact test when appropriate. The Kaplan-Meier method was used for survival function and compared using the log rank test. The $p$-value for statistical significance was defined as $\leq 0.05$. Statistical analysis was performed using Microsoft Excel (Microsoft Corporation, Redmond, WA) and IBM SPSS Statistics for Windows, version 23 (IBM Corporation, Armonk, NY).

\section{RESULTS}

The Dartmouth-Hitchcock interdisciplinary gastrointestinal tumor board saw 1,020 patients with pancreatic adenocarcinoma between 2004 and 2017. Of those cases, 742 had imaging available for retrospective AHPBA/SSAT/SSO resectability designation. Two hundred eleven patients had resectable pancreatic cancer, and 126 of those patients initiated neoadjuvant therapy. Of those 126 patients with resectable pancreatic adenocarcinoma who initiated neoadjuvant therapy, 108 patients completed treatment (86\%). Seventy-five of those patients underwent surgical exploration, with $100 \%$ completing surgical resection. Of those 75 patients, 39 received preoperative CRT only and 36 completed SCT and CRT in the preoperative setting. Of the 39 patients who underwent preoperative CRT, 11 were treated with gemcitabine alone and 28 were treated with gemcitabine plus cetuximab. Of the 36 patients who received SCT and CRT, 29 received gemcitabine plus docetaxel, six received gemcitabine/abraxane, and one received FOLFIRINOX.

TABLE 4 | Resection and survival outcomes in patients with resectable pancreatic adenocarcinoma.

Outcome Chemoradiation $\begin{gathered}\text { Chemotherapy \& } \\ \text { chemoradiation }\end{gathered} \quad p$-value

\begin{tabular}{|c|c|c|c|}
\hline \multicolumn{4}{|c|}{ RESECTION MARGINS (\%), $n=75$} \\
\hline Ro & $29(74)$ & $28(78)$ & \multirow[t]{2}{*}{0.73} \\
\hline R1 & $10(26)$ & $8(22)$ & \\
\hline \multicolumn{4}{|c|}{ OVERALL SURVIVAL AT 6 MONTHS (\%), $n=75$} \\
\hline Yes & $39(100)$ & $36(100)$ & \multirow[t]{2}{*}{1.00} \\
\hline No & $0(0)$ & $0(0)$ & \\
\hline \multicolumn{4}{|c|}{ OVERALL SURVIVAL AT 1 YEAR (\%), $n=75$} \\
\hline Yes & $36(92)$ & $33(92)$ & \multirow[t]{2}{*}{0.92} \\
\hline No & $3(8)$ & $3(8)$ & \\
\hline \multicolumn{4}{|c|}{ OVERALL SURVIVAL AT 3 YEAR (\%), $n=69$} \\
\hline Yes & $17(46)$ & $14(44)$ & \multirow[t]{2}{*}{0.86} \\
\hline No & $20(54)$ & $18(56)$ & \\
\hline \multicolumn{4}{|c|}{ RECURRENCE FREE SURVIVAL AT 6 MONTHS (\%), $n=75$} \\
\hline Yes & $39(100)$ & $36(100)$ & \multirow[t]{2}{*}{1.00} \\
\hline No & $0(0)$ & $0(0)$ & \\
\hline \multicolumn{4}{|c|}{ Recurrence Free Survival at 1 year (\%), $n=75$} \\
\hline Yes & $29(74)$ & $27(75)$ & \multirow[t]{2}{*}{0.95} \\
\hline No & $10(26)$ & $9(25)$ & \\
\hline \multicolumn{4}{|c|}{ RECURRENCE FREE SURVIVAL AT 3 YEARS (\%), $n=70$} \\
\hline Yes & $11(29)$ & $8(25)$ & \multirow[t]{2}{*}{0.71} \\
\hline No & $27(72)$ & $24(75)$ & \\
\hline
\end{tabular}

The borderline resectable pancreatic adenocarcinoma group consisted of 270 patients. One hundred ninety-four of those patients initiated neoadjuvant therapy. One hundred and forty-three patients out of the 194 patients who initiated neoadjuvant therapy completed treatment (74\%). Hundred patients underwent surgical exploration, and 74 of those patients completed surgical resection (74\%). Of those 74 patients who completed NAT and surgical resection, 34 patients received CRT alone and 40 completed SCT with CRT. Of the 34 patients who underwent preoperative CRT, six were treated with gemcitabine alone, and 28 were treated with gemcitabine plus cetuximab. Of the 40 patients who underwent SCT and CRT, 29 received gemcitabine plus docetaxel, nine received gemcitabine/abraxane, and two received FOLFIRINOX. Figure 1 maps the inclusion and progression of patients in this study. Figure 1A shows initial patient selection through Tumor Board and AHPBA/SSAT/SSO designation. Figure 1B outlines the progression of patients with resectable pancreatic adenocarcinoma through neoadjuvant therapy and surgical resection, and Figure 1C charts the progression of patients with borderline resectable pancreatic adenocarcinoma through neoadjuvant therapy and surgical resection. The follow up period for this study was 3 years, starting from date of diagnosis.

There was no difference in age, gender, endobiliary stent presence, tumor location, tumor diameter, $\mathrm{T}$ classification, $\mathrm{N}$ classification, or CA 19-9 between the two treatment groups in resectable or borderline resectable disease. These baseline characteristics are displayed in Table 2 for resectable pancreatic

TABLE 5 | Resection and survival outcomes in patients with borderline resectable pancreatic adenocarcinoma.

Outcome Chemoradiation $\begin{gathered}\text { Chemotherapy \& } \\ \text { chemoradiation }\end{gathered} \quad p$-value

\begin{tabular}{|c|c|c|c|}
\hline \multicolumn{4}{|c|}{ RESECTION MARGINS (\%), $n=74$} \\
\hline Ro & 23 (68) & $23(57)$ & \multirow[t]{2}{*}{0.37} \\
\hline R1 & $11(32)$ & 17 (43) & \\
\hline \multicolumn{4}{|c|}{ OVERALL SURVIVAL AT 6 MONTHS (\%), $n=74$} \\
\hline Yes & 34 (100) & $40(100)$ & \multirow[t]{2}{*}{1.00} \\
\hline No & $0(0)$ & $0(0)$ & \\
\hline \multicolumn{4}{|c|}{ OVERALL SURVIVAL AT 1 YEAR (\%), $n=74$} \\
\hline Yes & $29(85)$ & $37(93)$ & \multirow[t]{2}{*}{0.32} \\
\hline No & $5(15)$ & $3(8)$ & \\
\hline
\end{tabular}

OVERALL SURVIVAL AT 3 YEAR (\%), $n=69$

\begin{tabular}{|c|c|c|}
\hline Yes & $14(41)$ & $14(40)$ \\
\hline No & $20(59)$ & $21(60)$ \\
\hline
\end{tabular}

RECURRENCE FREE SURVIVAL AT 6 MONTHS (\%), $\boldsymbol{n}=74$

$\begin{array}{lccc}\text { Yes } & 33(97) & 40(100) & 0.46 \\ \text { No } & 1(3) & 0(0) & \\ \text { RECURRENCE FREE SURVIVAL AT } 1 \text { YEAR }(\%), \boldsymbol{n}=\mathbf{7 4} & \\ \text { Yes } & 19(56) & 29(73) & 0.14 \\ \text { No } & 15(44) & 11(27) & \end{array}$

RECURRENCE FREE SURVIVAL AT 3 YEARS (\%), $n=71$

$\begin{array}{llll}\text { Yes } & 9(26) & 11(30) & 0.76 \\ \text { No } & 25(74) & 26(70) & \end{array}$


adenocarcinoma and Table 3 for borderline resectable cancer. There was no significant difference in adjuvant therapy treatment following surgical resection in the two treatment groups for patients with resectable disease: $28 \%$ of patients who received CRT without SCT also received adjuvant therapy compared to $33 \%$ in patients who received neoadjuvant SCT with CRT $(p=0.65)$. There was no significant difference in patients who received adjuvant therapy following resection in borderline resectable cancer, with 22 and 34\% in the CRT alone and SCT with CRT treatment groups, respectively $(p=0.26)$.

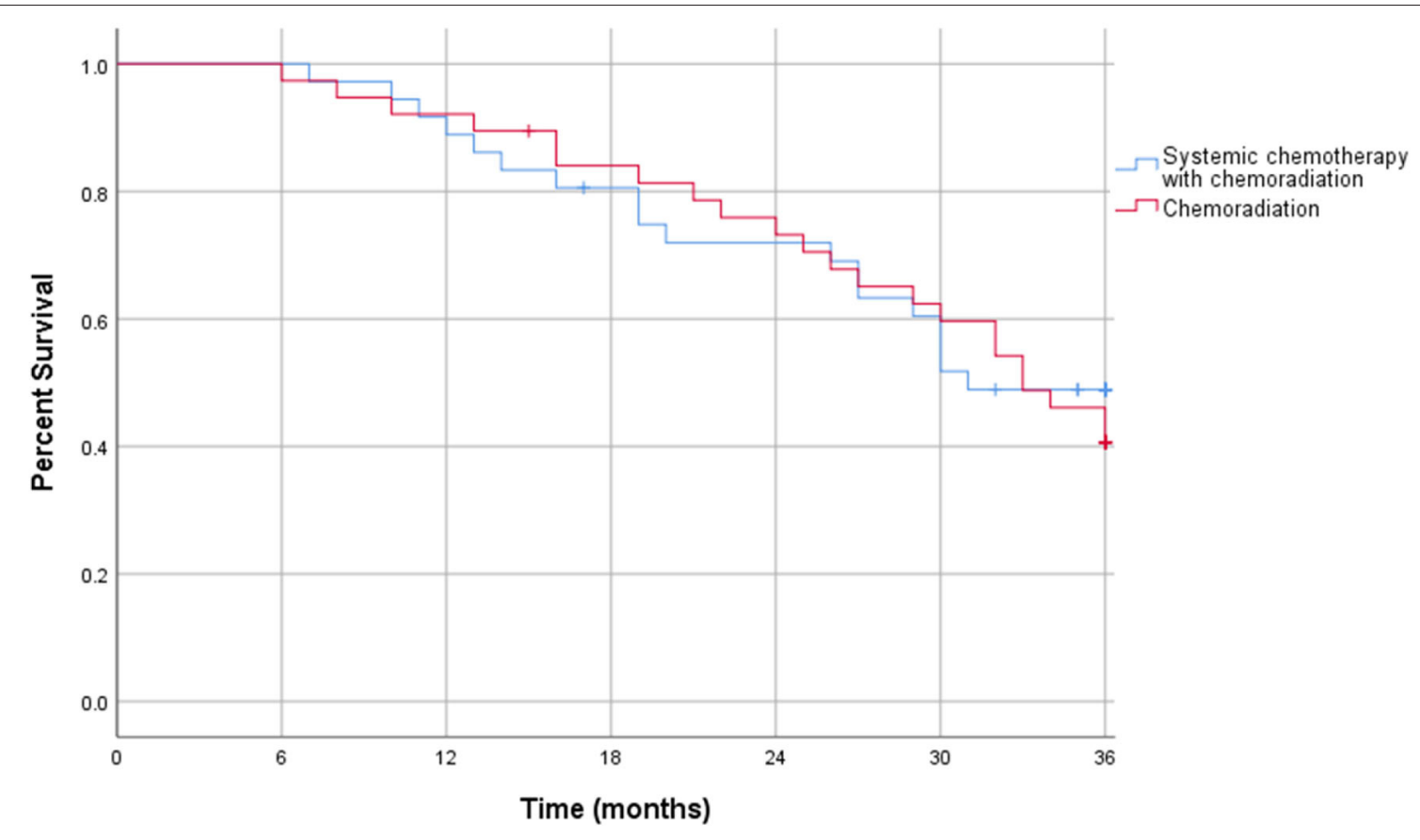

FIGURE 2 | Kaplan-Meier curve for resectable pancreatic adenocarcinoma.

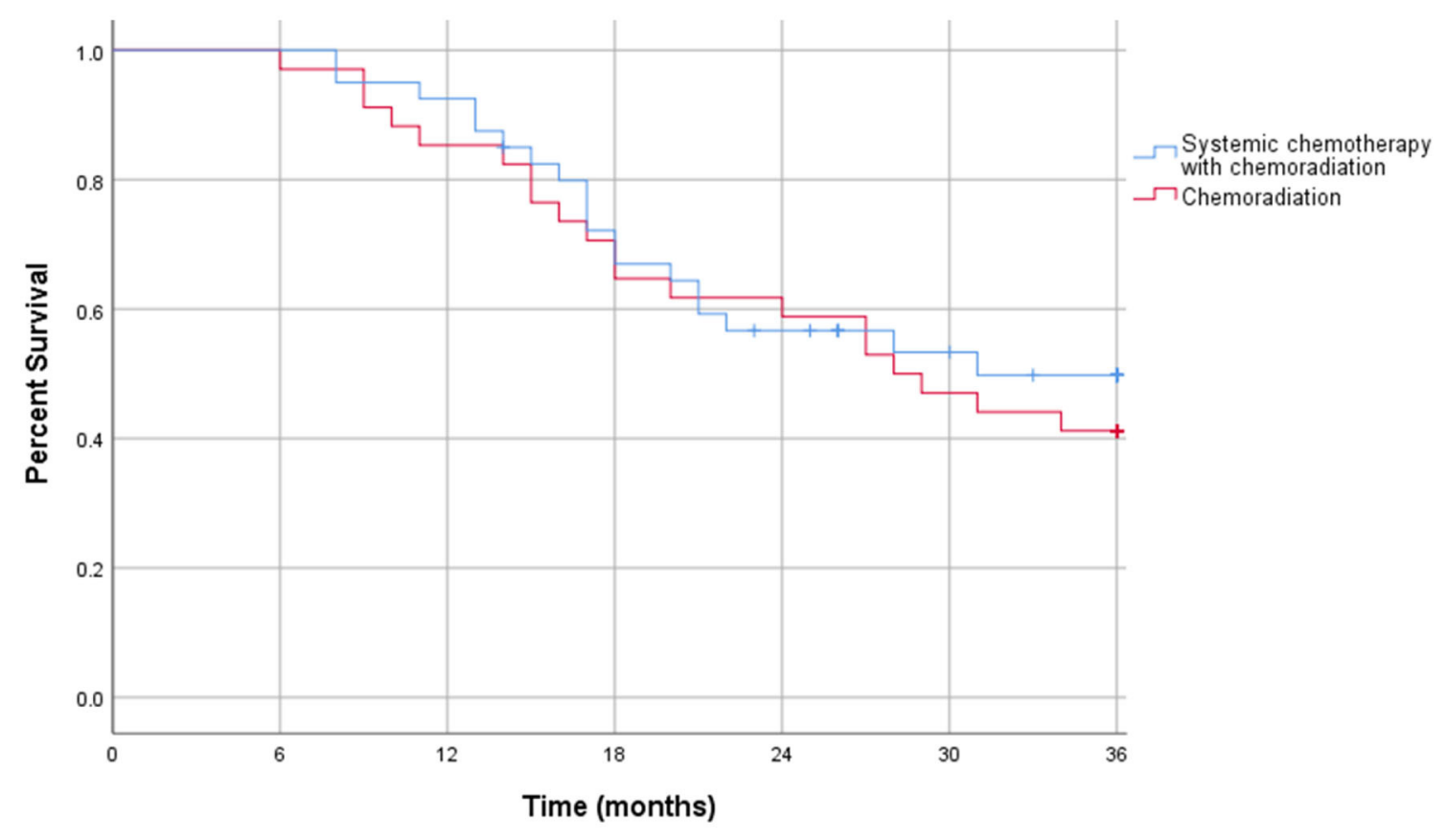

FIGURE 3 | Kaplan-Meier curve for borderline resectable pancreatic adenocarcinoma. 
TABLE 6 | Pattern of recurrence in patients with resectable pancreatic adenocarcinoma who completed neoadjuvant therapy and surgical resection.

\begin{tabular}{|c|c|c|c|}
\hline $\begin{array}{l}\text { Pattern of } \\
\text { recurrence }\end{array}$ & Chemoradiation & $\begin{array}{l}\text { Chemotherapy \& } \\
\text { Chemoradiation }\end{array}$ & $p$-value \\
\hline \multicolumn{4}{|c|}{ ANY RECURRENCE (\%), $n=75$} \\
\hline Yes & $27(69)$ & $21(58)$ & 0.37 \\
\hline No & $12(21)$ & $15(42)$ & \\
\hline \multicolumn{4}{|c|}{ LOCAL ONLY (\%), $n=74$} \\
\hline Yes & $5(13)$ & $1(3)$ & 0.11 \\
\hline No & $33(87)$ & $35(97)$ & \\
\hline \multicolumn{4}{|c|}{ DISTANT ONLY (\%), $n=74$} \\
\hline Yes & $16(42)$ & $16(44)$ & 0.84 \\
\hline No & $22(58)$ & $20(56)$ & \\
\hline \multicolumn{4}{|c|}{ BOTH LOCAL AND DISTANT (\%), $n=74$} \\
\hline Yes & $5(13)$ & $4(11)$ & 0.54 \\
\hline No & $33(87)$ & $32(89)$ & \\
\hline
\end{tabular}

In the resectable cancers, $74 \%$ of patients who received only CRT had R0 margins compared to $78 \%$ in the SCT with CRT group $(p=0.73)$. For both treatment groups, $92 \%$ of patents lived past 1 year $(p=0.92)$. There was no significant difference in overall 3 years survival: $46 \%$ of patients who received CRT alone survived at least 3 years compared to $44 \%$ in the SCT with CRT treatment group $(p=0.86)$. The recurrence free survival rates did not differ significantly between the treatment groups. At 1 year, $74 \%$ of patients achieved recurrence free survival in the CRT alone group compared to $75 \%$ in patients treated with SCT and CRT $(p=0.95) .29 \%$ of patients had recurrence free survival at 3 years in the CRT alone group compared to $25 \%$ in patients who received SCT with CRT $(p=0.71)$. The resection and survival outcomes for resectable pancreatic adenocarcinoma are displayed in Table 4.

In borderline resectable cancers, patients who received CRT alone had $68 \%$ R0 margins compared to $57 \%$ in patients who received SCT and CRT $(p=0.37)$. At 1 year, $85 \%$ of patients survived in the CRT alone group compared to $93 \%$ in patients treated with SCT and CRT $(p=0.32)$. There was no significant difference in overall 3 years survival: $41 \%$ of patients who received only neoadjuvant CRT survived at least 3 years compared to $40 \%$ in the SCT with CRT treatment group $(p=0.92) .56 \%$ of patients had recurrence free survival at 1 year in the CRT alone group compared to $73 \%$ in patients who received SCT with CRT $(p=0.14)$. The 3 years recurrence free survival was 26 and $30 \%$ for the CRT alone group and the SCT plus CRT group, respectively $(p=0.76)$. The resection and survival outcomes for borderline resectable pancreatic adenocarcinoma are displayed in Table 5.

The Kaplan-Meier curve for resectable pancreatic adenocarcinoma showed no difference in the survival function up to 3 years between the two treatment groups, with a mean survival of 28.19 months for SCT with CRT and 28.93 for CRT alone $(p=0.87)$ (Figure 2$)$. For borderline resectable adenocarcinoma, the Kaplan-Meier curve also showed no difference in the survival function between the two treatment
TABLE 7 | Pattern of recurrence in patients with borderline resectable pancreatic adenocarcinoma who completed neoadjuvant therapy and surgical resection.

\begin{tabular}{|c|c|c|c|}
\hline $\begin{array}{l}\text { Pattern of } \\
\text { recurrence }\end{array}$ & Chemoradiation & $\begin{array}{l}\text { Chemotherapy \& } \\
\text { chemoradiation }\end{array}$ & $p$-value \\
\hline \multicolumn{4}{|c|}{ ANY RECURRENCE (\%), $n=74$} \\
\hline Yes & $25(74)$ & $30(75)$ & 0.89 \\
\hline No & $9(26)$ & $10(25)$ & \\
\hline \multicolumn{4}{|c|}{ Local ONLY (\%), $n=74$} \\
\hline Yes & $5(15)$ & $1(3)$ & 0.067 \\
\hline No & $29(85)$ & $39(97)$ & \\
\hline \multicolumn{4}{|c|}{ DISTANT ONLY (\%), $n=74$} \\
\hline Yes & $15(44)$ & $16(40)$ & 0.72 \\
\hline No & $19(56)$ & $24(60)$ & \\
\hline \multicolumn{4}{|c|}{ BOTH LOCAL AND DISTANT (\%), $n=74$} \\
\hline Yes & $5(15)$ & $4(10)$ & 0.34 \\
\hline No & $29(8)$ & $36(90)$ & \\
\hline
\end{tabular}

TABLE 8 | Intention to treat survival outcomes in patients with resectable pancreatic adenocarcinoma.

\begin{tabular}{|c|c|c|c|}
\hline Outcome & Chemoradiation & $\begin{array}{l}\text { Chemotherapy \& } \\
\text { chemoradiation }\end{array}$ & $p$-value \\
\hline \multicolumn{4}{|c|}{ OVERALL SURVIVAL AT 6 MONTHS (\%), $n=114$} \\
\hline Yes & $52(46)$ & $54(47)$ & 0.96 \\
\hline No & $4(4)$ & $4(4)$ & \\
\hline \multicolumn{4}{|c|}{ OVERALL SURVIVAL AT 1 YEAR (\%), $n=112$} \\
\hline Yes & $43(38)$ & $41(37)$ & 0.66 \\
\hline No & $13(12)$ & $15(13)$ & \\
\hline \multicolumn{4}{|c|}{ OVERALL SURVIVAL AT 3 YEAR (\%), $n=106$} \\
\hline Yes & $18(17)$ & $15(14)$ & 0.62 \\
\hline No & $36(34)$ & $37(35)$ & \\
\hline
\end{tabular}

groups, with a mean survival of 26.83 months for SCT with CRT and 25.94 for CRT alone $(p=0.58)$ (Figure 3). There was no significant difference in recurrence patterns (local, distant, or both) between the treatment groups in either resectable or borderline resectable pancreatic adenocarcinoma (Tables 6, 7 , respectively).

Additional survival analysis was done using intention to treat methodology and included all patients who initiated neoadjuvant therapy, regardless of neoadjuvant therapy completion or surgical resection. In the intention to treat survival analysis for resectable pancreatic adenocarcinoma, there was no significant difference in 1 year overall survival: $38 \%$ of patients who received CRT alone compared to $37 \%$ in the SCT with CRT treatment group $(p=0.66)$. There was also no difference in 3 years overall survival with $17 \%$ in CRT patients and $14 \%$ in SCT with CRT patients $(p=0.62)$. These outcomes are displayed in Table 8. The intention to treat Kaplan-Meier curve for resectable pancreatic adenocarcinoma showed no difference in the survival function up to three years between the two treatment groups, with a mean survival of 22.32 months for SCT with CRT and 23.81 for CRT alone $(p=0.89)$ (Figure 4$)$. 


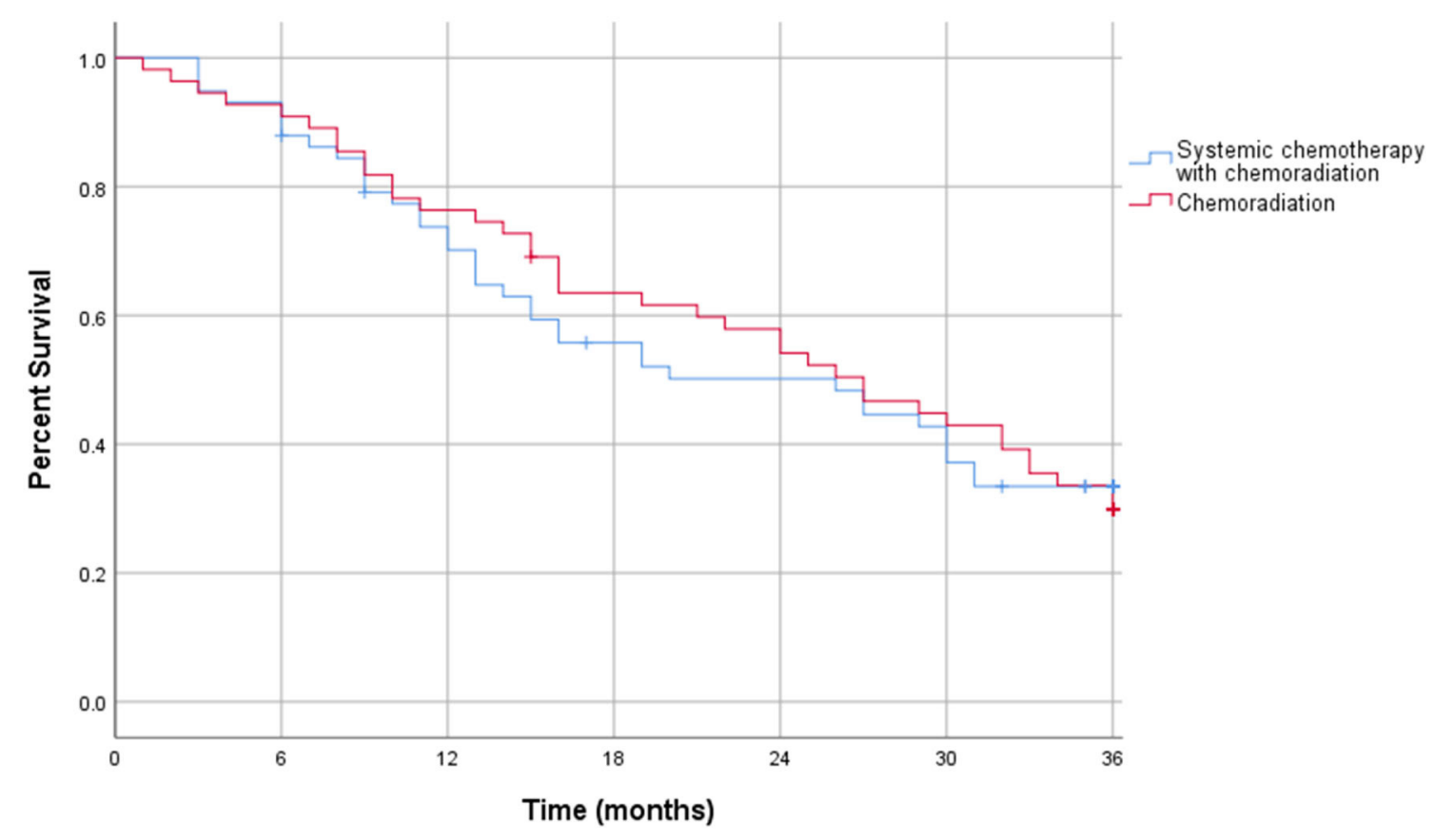

FIGURE 4 | Intention to treat Kaplan-Meier curve for resectable pancreatic adenocarcinoma.

TABLE 9 | Intention to treat survival outcomes in patients with borderline resectable pancreatic adenocarcinoma.

\begin{tabular}{|c|c|c|c|}
\hline Outcome & Chemoradiation & $\begin{array}{l}\text { Chemotherapy \& } \\
\text { chemoradiation }\end{array}$ & $p$-value \\
\hline \multicolumn{4}{|c|}{ OVERALL SURVIVAL AT 6 MONTHS (\%), $n=172$} \\
\hline Yes & $67(39)$ & $87(51)$ & 0.16 \\
\hline No & $11(6)$ & $7(4)$ & \\
\hline \multicolumn{4}{|c|}{ OVERALL SURVIVAL AT 1 YEAR (\%), $n=171$} \\
\hline Yes & $58(34)$ & $62(36)$ & 0.27 \\
\hline No & $20(12)$ & $31(18)$ & \\
\hline \multicolumn{4}{|c|}{ OVERALL SURVIVAL AT 3 YEARS (\%), $n=164$} \\
\hline Yes & $15(9)$ & $20(12)$ & 0.53 \\
\hline No & 63 (38) & $66(40)$ & \\
\hline
\end{tabular}

The intention to treat survival analysis for borderline resectable pancreatic adenocarcinoma demonstrated no significant difference in overall all survival rate. At 1 year, $34 \%$ of patients survived in the CRT alone group compared to $36 \%$ in patients treated with SCT and CRT ( $\mathrm{p}=0.27$ ). At 3 years post diagnosis, there was a $9 \%$ survival rate in the CRT alone group compared to $12 \%$ in patients treated with SCT and CRT $(p=0.53)$. The intention to treat survival outcomes for borderline resectable pancreatic adenocarcinoma are displayed in Table 9. Additionally, the intention to treat Kaplan-Meier curve for borderline resectable disease showed no difference in the survival function between the two treatment groups, with a mean survival of 19.99 months for SCT with CRT and 17.08 for CRT alone $(p=0.19)$ (Figure 5).

\section{DISCUSSION}

While the efficacy of neoadjuvant therapy in localized pancreatic adenocarcinoma is still highly debated, neoadjuvant therapy is increasingly being utilized at institutions across the world. With modern systemic chemotherapy, oncologic outcomes in clinical trials show improved overall survival benefits not only in metastatic disease, but in localized cancer as well $(7-9,13,14)$. In addition to defining the role of neoadjuvant therapy, more insight is needed on what the optimal treatment regimen is and whether that includes SCT, CRT, or both. The existing data comparing CRT alone and SCT plus CRT in locally advanced disease is contradictory: in comparing SCT with and without CRT, Hugeut et al. found survival benefits in patients who received SCT with CRT, while Hammal et al. saw no improvement in survival in patients who received SCT and CRT $(15,16)$. While multiple trials have compared different regimens and combinations of SCT, CRT, or both in resectable and borderline resectable pancreatic adenocarcinoma, there is still more work to elucidate the optimal treatment regimen (17-21).

This study reports 13 years of experience using neoadjuvant therapy for pancreatic adenocarcinoma with regimens including SCT with CRT, and CRT alone. The majority of patients with resectable and borderline pancreatic carcinoma who initiated neoadjuvant therapy completed their regimens and made it to surgical resection. We compared margin negative resection rates as well as recurrence patterns and saw no difference between the two treatment groups in either resectable or borderline resectable disease. We also did not find any significant advantage in overall or recurrence free survival between patients treated with SCT plus CRT vs. CRT alone. Our intention to treat survival analysis 


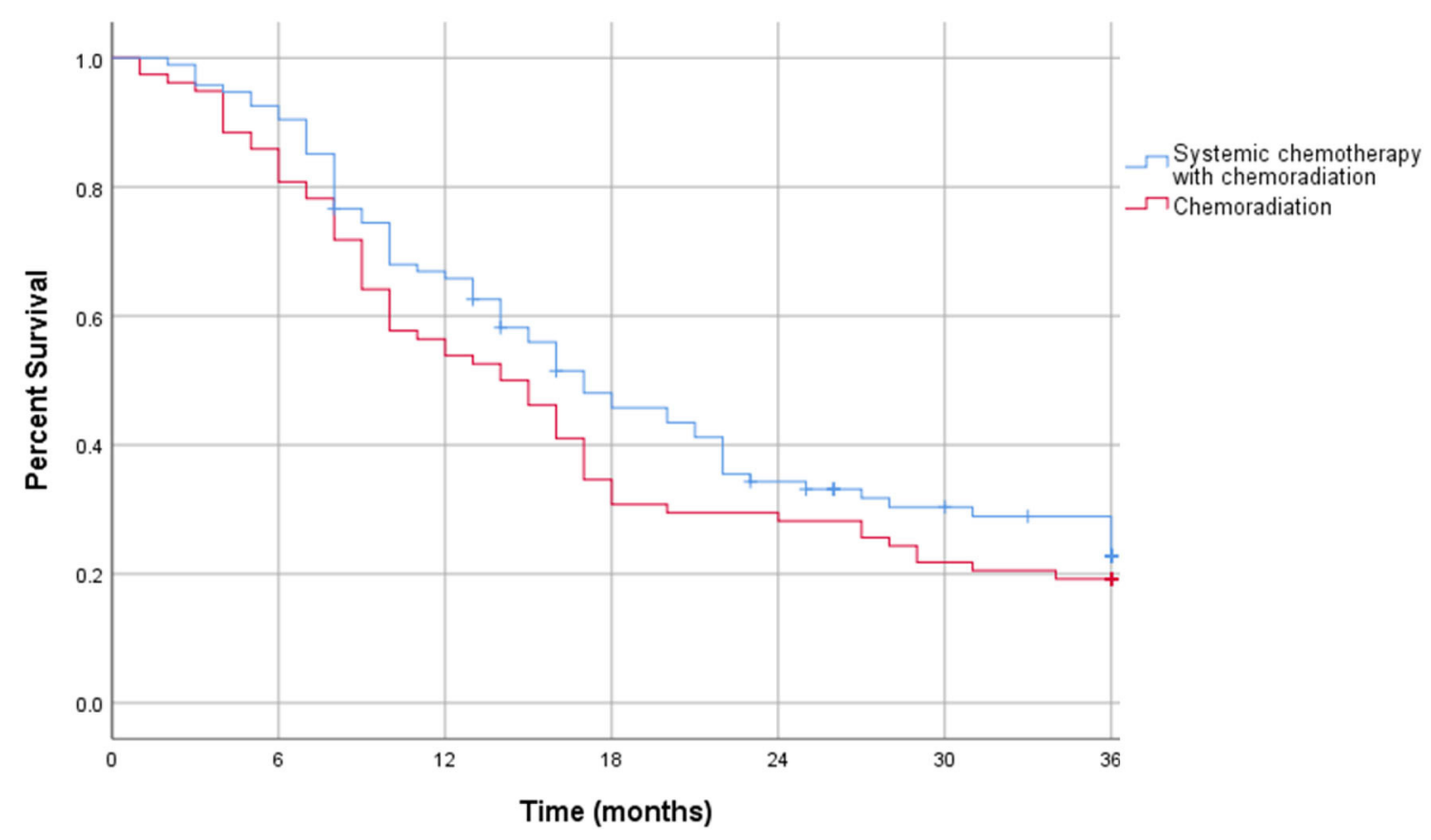

FIGURE 5 | Intention to treat Kaplan-Meier curve for borderline resectable pancreatic adenocarcinoma.

was consistent with our findings from examining only patients who achieved surgical resection.

Our findings suggest that for resectable and borderline resectable pancreatic adenocarcinoma, SCT with CRT does not offer any survival or recurrence benefits compared to CRT alone. This brings into question whether neoadjuvant SCT is necessary if neoadjuvant CRT alone is just as effective. SCT with CRT not only increases the delay in surgical resection compared to CRT alone, but it also subjects patients to increased side effects and drug toxicity (4). An alternative treatment approach to consider is using neoadjuvant CRT followed by surgical resection and systemic adjuvant therapy. This approach may provide patients with the benefits of neoadjuvant therapy, while decreasing further delay in potentially curative resection and offering the benefits of systemic therapy. A clinical trial is needed to provide insight on whether SCT in the neoadjuvant or adjuvant setting provides more benefits in patients receiving neoadjuvant CRT with resectable and borderline resectable pancreatic adenocarcinoma.

This study has several limitations. First, it is limited by its sample size and non-randomized observational design. The type of neoadjuvant therapy a patient received was made on an individual basis by the treating physicians. There was no over-arching guide, and it is possible that patients with more adverse clinical features were preferentially given one type of therapy over another, as this was not controlled for in our analysis. Additionally, grouping the different regimens into SCT with CRT vs. CRT alone does not allow us to compare each individual regimen directly. The heterogeneity in the SCT with CRT group includes the currently popular systemic regimens of FOLFIRINOX and gemcitabine/abraxane as well as old regimens no longer commonly used. This may account for the lack of benefit seen over CRT alone, as recent studies focusing on these newer regimens have shown promising results in borderline and locally advanced disease, and trials in resectable disease are beginning to shed more light (22-25). Although intention to treat survival analysis was done, some of our other data analysis excluded patients who were not surgically resected. This number was similar in both treatment groups for both resectable and borderline resectable cancer however, and was done to allow analysis of variables such as resection margins and recurrence patterns. Lastly, the follow up time of this study limits the detection of a difference in the overall and recurrence free survival beyond 3 years.

In conclusion, both SCT with CRT and CRT alone are effective options for neoadjuvant therapy in resectable and borderline resectable pancreatic adenocarcinoma. Our findings bring into question whether SCT is necessary to increase survival benefits if neoadjuvant CRT alone is just as effective. This argues for the need for better systemic therapy to justify its use, or whether neoadjuvant CRT alone should be used with adjuvant SCT following resection. While there is need for a prospective randomized clinical trial, our study is an early attempt at elucidating the effects of neoadjuvant SCT with CRT compared to CRT alone in resectable and borderline resectable pancreatic adenocarcinoma.

\section{DATA AVAILABILITY STATEMENT}

The raw data supporting the conclusions of this article will be made available by the authors, without undue reservation, to any qualified researcher. 


\section{ETHICS STATEMENT}

The studies involving human participants were reviewed and approved by Dartmouth Committee for the Protection of Human Subjects. The patients/participants provided their written informed consent to participate in this study.

\section{REFERENCES}

1. Cronin KA, Lake AJ, Scott S, Sherman RL, Noone AM, Howlader N, et al. Annual report to the nation on the status of cancer, part i: national cancer statistics. Cancer. (2018) 124:2785-800. doi: 10.1002/cncr.31551

2. Russo S, Ammori J, Eads J, Dorth J. The role of neoadjuvant therapy in pancreatic cancer: a review. Fut Oncol. (2016) 12:669-85. doi: $10.2217 /$ fon. 15.335

3. Cloyd JM, Katz MH, Prakash L, Varadhachary GR, Wolff RA, Shroff RT, et al. Preoperative therapy and pancreatoduodenectomy for pancreatic ductal adenocarcinoma: a 25-year single-institution experience. J Gastrointest Surg. (2017) 21:164-74. doi: 10.1007/s11605-016-3265-1

4. Heinrich N, Lang H. Neoadjuvant therapy of pancreatic cancer: definitions and benefits. Int J Mol Sci. (2017) 18:1622. doi: 10.3390/ijms18081622

5. Russo S, Saif MW. Neoadjuvant therapy for pancreatic cancer: an ongoing debate. Therap Adv Gastroenterol. (2016) 9:429-36. doi: 10.1177/1756283X16646524

6. Abrams RA, Lowy AM, O’Reilly EM, Wolff RA, Picozzi VJ, Pisters PWT. Combined modality treatment of resectable and borderline resectable pancreas cancer: expert consensus statement. Ann Surg Oncol. (2009) 16:1751-6. doi: 10.1245/s10434-009-0413-9

7. Zhan HX, Xu JW, Wu D, Wu ZY, Wang L, Hu SY, et al. Neoadjuvant therapy in pancreatic cancer: a systematic review and meta-analysis of prospective studies. Cancer Med. (2017) 6:1201-19. doi: 10.1002/cam4.1071

8. Coveler AL, Herman JM, Simeone DM, Chiorean EG. Pancreatic cancer: multidisciplinary management. Am Soc Clin Oncol Educ Book. (2016) 35:e217-26. doi: 10.1200/EDBK_160827

9. Greer SE, Pipas JM, Sutton JE, Zaki BI, Tsapakos M, Colacchio TA, et al. Effect of neoadjuvant therapy on local recurrence after resection of pancreatic adenocarcinoma. J Am Coll Surg. (2008) 206:451-7. doi: 10.1016/j.jamcollsurg.2007.10.002

10. Conroy T, Desseigne F, Ychou M. FOLFIRINOX versus gemcitabine for metastatic pancreatic cancer. N Engl J Med. (2011) 364:1817-25. doi: 10.1056/NEJMoa1011923

11. Von Hoff DD, Ervin T, Arena FP, Chiorean EG, Infante J, Moore M, et al. Increased survival in pancreatic cancer with nab-paclitaxel plus gemcitabine. N Engl J Med. (2013) 369:1691-703. doi: 10.1056/NEJMoa1304369

12. Jang JY, Han Y, Lee H, Kim SW, Kwon W, Lee KH, et al. Oncological benefits of neoadjuvant chemoradiation with gemcitabine versus upfront surgery in patients with borderline resectable pancreatic cancer: a prospective, randomized, open-label, multicenter phase 2/3 trial. Ann Surg. (2018) 268:215-22. doi: 10.1097/SLA.0000000000002705

13. Evans DB, Varadhachary GR, Crane CH, Sun CC, Lee JE, Pisters PW, et al. Preoperative gemcitabine-based chemoradiation for patients with resectable adenocarcinoma of the pancreatic head. J Clin Oncol. (2008) 26:3496-502. doi: 10.1200/JCO.2007.15.8634

14. Dhir M, Malhotra GK, Sohal DPS, Hein NA, Smith LM, O’Reilly EM, et al. Neoadjuvant treatment of pancreatic adenocarcinoma: a systematic review and meta-analysis of 5520 patients. World J Surg Oncol. (2017) 15:183. doi: 10.1186/s12957-017-1240-2

15. Huguet F, André T, Hammel P, Artru P, Balosso J, Selle F, et al. Impact of chemoradiotherapy after disease control with chemotherapy in locally advanced pancreatic adenocarcinoma in GERCOR phase II and III studies. J Clin Oncol. (2007) 25:326-31. doi: 10.1200/JCO.2006.07.5663
AUTHOR CONTRIBUTIONS

KT, DF, TG, and KS contributed to the study concept, design, acquisition of data, analysis, interpretation, drafting, and editing of the manuscript, and critical revision. All authors contributed to the article and approved the submitted version.

16. Hammel P, Huguet F, van Laethem JL, Goldstein D, Glimelius B, Artru P, et al. Effect of chemoradiotherapy vs chemotherapy on survival in patients with locally advanced pancreatic cancer controlled after 4 months of gemcitabine with or without erlotinib: the LAP07 randomized clinical trial. JAMA. (2016) 315:1844-53. doi: 10.1001/jama.2016.4324

17. Dhir M, Zenati MS, Hamad A, Singhi AD, Bahary N, Hogg ME, et al. FOLFIRINOX versus gemcitabine/nab-paclitaxel for neoadjuvant treatment of resectable and borderline resectable pancreatic head adenocarcinoma. Ann Surg Oncol. (2018) 25:1896-903. doi: 10.1245/s10434-0186512-8

18. Chapman BC, Gleisner A, Rigg D, Messersmith W, Paniccia A, Meguid $\mathrm{C}$, et al. Perioperative and survival outcomes following neoadjuvant FOLFIRINOX versus gemcitabine abraxane in patients with pancreatic adenocarcinoma. JOP. (2018) 19:75-85.

19. Berriochoa CA, Abdel-Wahab M, Leyrer CM, Khorana A, Matthew Walsh R, Kumar AM. Neoadjuvant chemoradiation for non-metastatic pancreatic cancer increases margin-negative and node-negative rates at resection. J Dig Dis. (2017) 18:642-9. doi: 10.1111/1751-2980.12551

20. Takahashi S, Kinoshita T, Konishi M, Gotohda N, Kato Y, Kinoshita $\mathrm{T}$, et al. Borderline resectable pancreatic cancer: rationale for multidisciplinary treatment. J Hepatobiliary Pancreat Sci. (2011) 18:567-74. doi: 10.1007/s00534-011-0371-z

21. Rose JB, Rocha FG, Alseidi A, Biehl T, Moonka R, Ryan JA, et al. Extended neoadjuvant chemotherapy for borderline resectable pancreatic cancer demonstrates promising postoperative outcomes and survival. Ann Surg Oncol. (2014) 21:1530-7. doi: 10.1245/s10434-014-3486-Z

22. Pouypoudat C, Buscail E, Cossin S, Cassinotto C, Terrebonne E, Blanc JF, et al. FOLFIRINOX-based neoadjuvant chemoradiotherapy for borderline and locally advanced pancreatic cancer: a pilot study from a tertiary centre. Dig Liver Dis. (2019) 51:1043-9. doi: 10.1016/j.dld.2019.03.004

23. Wagner M, Antunes C, Pietrasz D, Cassinotto C, Zappa M, Sa Cunha A, et al. CT evaluation after neoadjuvant FOLFIRINOX chemotherapy for borderline and locally advanced pancreatic adenocarcinoma. Eur Radiol. (2017) 27:310416. doi: 10.1007/s00330-016-4632-8

24. Napolitano F, Formisano L, Giardino A, Girelli R, Servetto A, Santaniello $A$, et al. Neoadjuvant treatment in locally advanced pancreatic cancer (LAPC) patients with folfirinox or gemcitabine nabpaclitaxel: a singlecenter experience and a literature review. Cancers. (2019) 11:981. doi: 10.3390/cancers11070981

25. Oba A, Ho F, Bao QR, Al-Musawi MH, Schulick RD, Del Chiaro M. Neoadjuvant treatment in pancreatic cancer. Front Oncol. (2020) 10:245. doi: $10.3389 /$ fonc. 2020.00245

Conflict of Interest: The authors declare that the research was conducted in the absence of any commercial or financial relationships that could be construed as a potential conflict of interest.

Copyright (C) 2020 Trinh, Fischer, Gardner and Smith. This is an open-access article distributed under the terms of the Creative Commons Attribution License (CC BY). The use, distribution or reproduction in other forums is permitted, provided the original author(s) and the copyright owner(s) are credited and that the original publication in this journal is cited, in accordance with accepted academic practice. No use, distribution or reproduction is permitted which does not comply with these terms. 